Academic City University College - Accra Ghana

Society for Multidisciplinary \& Advanced Research Techniques (SMART) Africa

Tony Blair Institute for Global Change

FAIR Forward - Artificial Intelligence for All - Deutsche Gesellschaft für Internationale Zusammenarbeit (GIZ) GmbH

Accra Bespoke Multidisciplinary Innovations Conference (ABMIC)

\& The Africa Al Stakeholders' Summit

$14^{\text {th }}$ December, 2021

\title{
Autonomous Road Crossing Surveillance System
}

Daniel Antwi Mensah \& Emmanuel Djaba

Ghana Institute of Management and Public Administration (GIMPA)

Accra, Ghana

E-mail: mensahhd78@gmail.com

Phone: +233558782261 


\title{
Autonomous Road Crossing Surveillance System
}

\author{
Daniel Antwi Mensah \& Emmanuel Djaba
}

\begin{abstract}
Accidents related with vulnerable pedestrians around crosswalks are continued so that proactive safety support system is required. Pedestrian detection from frames captured by a camera is a significant and yet challenging task. An autonomous road crossing surveillance system would be ideal for tracking pedestrians who want to cross the road and assist them. A practical solution for aiding pedestrians regularly, a road crossing surveillance with real-time Pedestrian Detection. Since the background subtraction from videos and images is still a persistent problem and difficult to accomplish. A Haar Cascade Classifier with the full-body detection algorithm is used to detect people in real-time captured by a camera.
\end{abstract}

Keywords: Pedestrian crossing, surveillance, road crossing surveillance, surveillance system, autonomous, Haar Cascade Classifier.

\section{BACKGROUND TO THE STUDY}

A pedestrian crossing is a place where pedestrians can cross a street and where motorists must stop to let them cross. In many places, the road network is constructed without considering the safety of pedestrians, especially children. Pedestrian's safety is reliant primarily on people making appropriate decisions when crossing the street, as well as necessity for drivers to be watchful and thoughtful. A pedestrian, as defined for this research paper, is any individual on foot, walking, running, jogging, or hiking who is involved in a motor vehicle crash or accident (Levulytė et al., 2017).

\subsection{Statement of Problem}

Pedestrians are amongst the most exposed road users due to a inadequate pedestrian crossing amenities. (Glèlè-ahanhanzo et al., 2021). The trend of fast urbanization and economic progress has resulted in an unprecedented increase in vehicle numbers around the world. The growth in vehicle numbers has resulted in a huge number of collisions and places a lot of strain on the current road system. (Jing et al., 2017). Pedestrians also has to wait for some specific time before getting to cross the road while cars will also have to wait in pedestrian traffic even if there are no pedestrians. This is seen as some kind of time wasting of both the pedestrians and the drivers.

\subsection{Objective}

The purpose of this study is to propose, design, develop and implement an autonomous road crossing surveillance system that will help individuals in the crossing of roads safely in their everyday lives. 


\section{METHODOLOGY}

\subsection{The Research Design}

This research uses the design science methodology in tackling this problem. The Design Science Methodology is defined as a standard that aims to create inventions that clarify the ideas, processes, technological capacities, and products that may be used to efficiently evaluate, design, implement, manage, and utilize information systems (Bisandu, 2016). Designing, creating, and assessing instructional programs, procedures, and products that must meet internal reliability and effectiveness standards. (Richey \& Klein, 2014). After the problem has been well understood, the development phase begins.

It entails putting the tentative design into action, resulting in the artifact. At this point, a prototype may be required to determine whether the proposed solution is a viable solution to the problem. A prototype is a pre-production sample or release of a real product that is used to validate a concept or development process. A prototype's objective is to provide a tangible representation of solutions to problems that have previously been described and discussed during the problem identification stage. We have as of late, acknowledged the utilization of interpretive research ideal models, yet the subsequent research output is still mostly explanatory and it very well may be contended, not frequently applied to the arrangement and solution to issues experienced in term and usage (Peffers et al., 2007).

Here, availability of components, safety, and usability are very important derails one must look out for. In the development of the suggested solution, the Extreme Programming (XP) method of development under the Agile Development Methodology will be used. This software development methodology was selected because it ensures iterative development and testing throughout the project's software development lifecycle. Continuous iteration defines a process or a set of processes in iterative software development such as integration, testing, delivery, and deployment. Development and testing are carried out concurrently in the Agile Methodology.

\subsection{The Solution of the Problem}

The Road Crossing Surveillance System is a Machine Learning Artificial Intelligence software that aid pedestrians to cross roads safely. The objective of this software is to reduce pedestrian waiting time and accidents when it comes to the crossing of roads. This research is meant to arguably solve the problems of both the pedestrian crossing the road and the driver using the road. The traffic light will turn red for drivers to stop only if there are a certain number of pedestrians ready to cross the road and therefore, if there are no pedestrians to cross the road, the traffic light turns green for the oncoming traffic(drivers). This is said to reduce waiting time for both drivers and pedestrians at the pedestrian crossing and if implemented well, will also reduce accidents on these pedestrian crossing. Solving this problem will help decrease the rate at which pedestrian accidents occur when they are crossing roads and also minimize the waiting time of pedestrians on the roadside waiting to cross the road

\section{IMPLEMENTATION}

The Road Crossing Surveillance System will use a machine-learning algorithm to detect pedestrians in a video feed. The system detects, tracks and counts pedestrians identified in the video feed. Once the system identifies $(x)$ number of pedestrians, the system passes a command to stop oncoming vehicles only when pedestrians were found in the video feed. If not, the system will pass a greenlight for vehicles to keep moving. 
In this case, there would be two sets of traffic lights (one facing the oncoming traffic and the other one facing the pedestrians) which will be mounted and connected to a control device. There will be a camera also attached to the mounted traffic lights and also connected to this control device. The control device will now act as the communication medium between what signal the camera sends and what action the traffic lights could carry out.

When the camera detects $(x)$ number of pedestrians, the control device will issue a stop command for the oncoming traffic and also issue a green light command for pedestrians to cross. When the scene is clear, the control device will issue a stop command for the pedestrian light and also issue a green light command for the oncoming traffic.

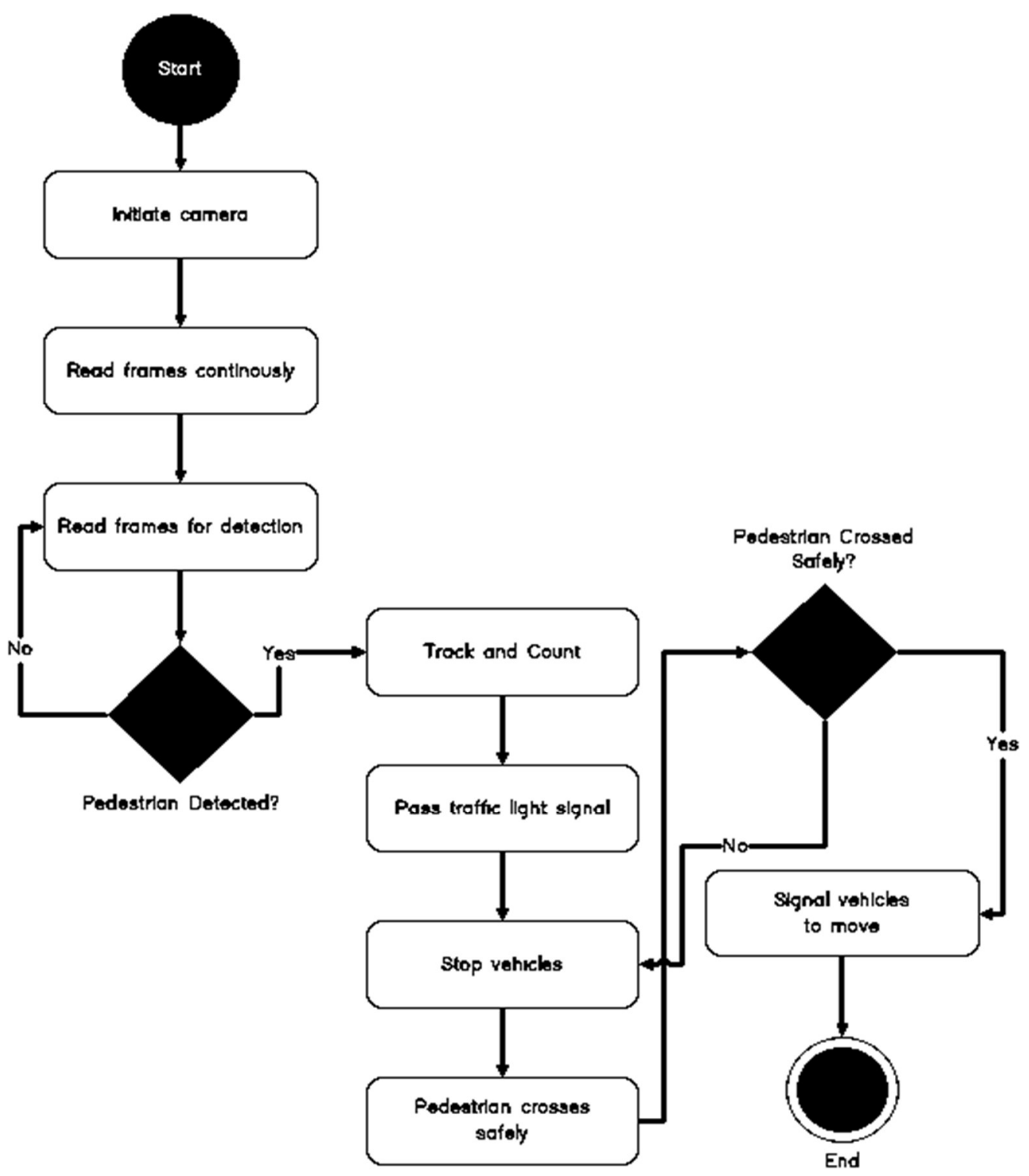

Fig 1: An Activity Diagram of the Proposed System 


\section{Video Capturing and Frame Reading}

A video feed is first captured from a camera with the help of OpenCV technology. The video feed is then read frame-by-frame into the code segment. The code segment loops through the video feed reading every frame until the video feed is terminated.

\section{Pedestrian Detection}

The detection of people is achieved with the help of the Haar Cascade Classifier technique in the OpenCV library. The Haar Cascade Classifier uses a full-body detection classifier to detect people in the looping frames of the video feed. The classifier will also read and examine all frames of the video feed that has been captured by the previous code segment for detection to take place.

\section{Showing Detection Onscreen}

A code segment is written to draw rectangles around the detected humans to show that detections are taking place. The code segment communicates with the Haar Cascades Classifier as detections are ongoing to demonstrate the detection of pedestrians.

\section{Counting of Pedestrians Detected Onscreen}

As detections are taking place, detected pedestrians are also being counted to help know how many humans are on screen. The number of detected pedestrians is displayed onscreen.

\section{Passing Command to Traffic Light Signals}

When the camera captures frames and detection takes place, the code segment then passes a traffic light signal command telling oncoming cars that a pedestrian wants to cross the road. The traffic light stops the cars allowing the pedestrian to cross the road safely.

\section{Functional Requirements}

- The camera should be able to be started by the system

- The system should be able to read the camera's live video stream.

- The system should be able to convert the camera's live video stream into frames.

- In the video feed, the system should be able to detect humans.

- The system should be able to count the number of humans that are detected in the video.

- The system should be able to signal cars to stop upon detection.

\section{Non-Functional Requirements}

- Security: The system should be protected against attacks

- Portability: The system should be able to work in different environments

- Responsiveness: The system should be quick to respond to commands.

- Usability: The system should be as user-friendly as possible.

- Reliability: The system should be able to run for a given period without failure.

- Availability: The system should be accessible for operation during some period.

- Maintainability: The system should support a short maintenance duration. 
Technology Stack

Python

Open-CV library

Raspberry Pi

Night Vision/Dark Fighter outdoor camera

\section{EVALUATION OF THE AUTONOMOUS ROAD CROSSING SYSTEM}

To evaluate the proposed constraints for the autonomous road crossing system, several testing and experiments were carried out. Frames of a video, whether captured by a camera or an existing video are read into the system for detection to start. Earlier, the proposed system was tested using the Histogram of Oriented Gradients detection method running on a machine with an Intel Core i7-7700HQ and 16GB of RAM. The Histogram of Oriented gradient detection method was not doing a good job at detecting two people walking together. It tends to detect multiple people walking together as a single entity.The Harr Cascade Classifier on the other hand, running on the same machine did a great job at detecting multiple people whether they are standing still or walking.

\subsection{Experimental Results}

The figures below show detection results using the pre-trained model acquired from the Haar Cascade Classifier. The result shows that for higher detection ratio, real dataset which is captured in real environment and passed to the pre-trained model.
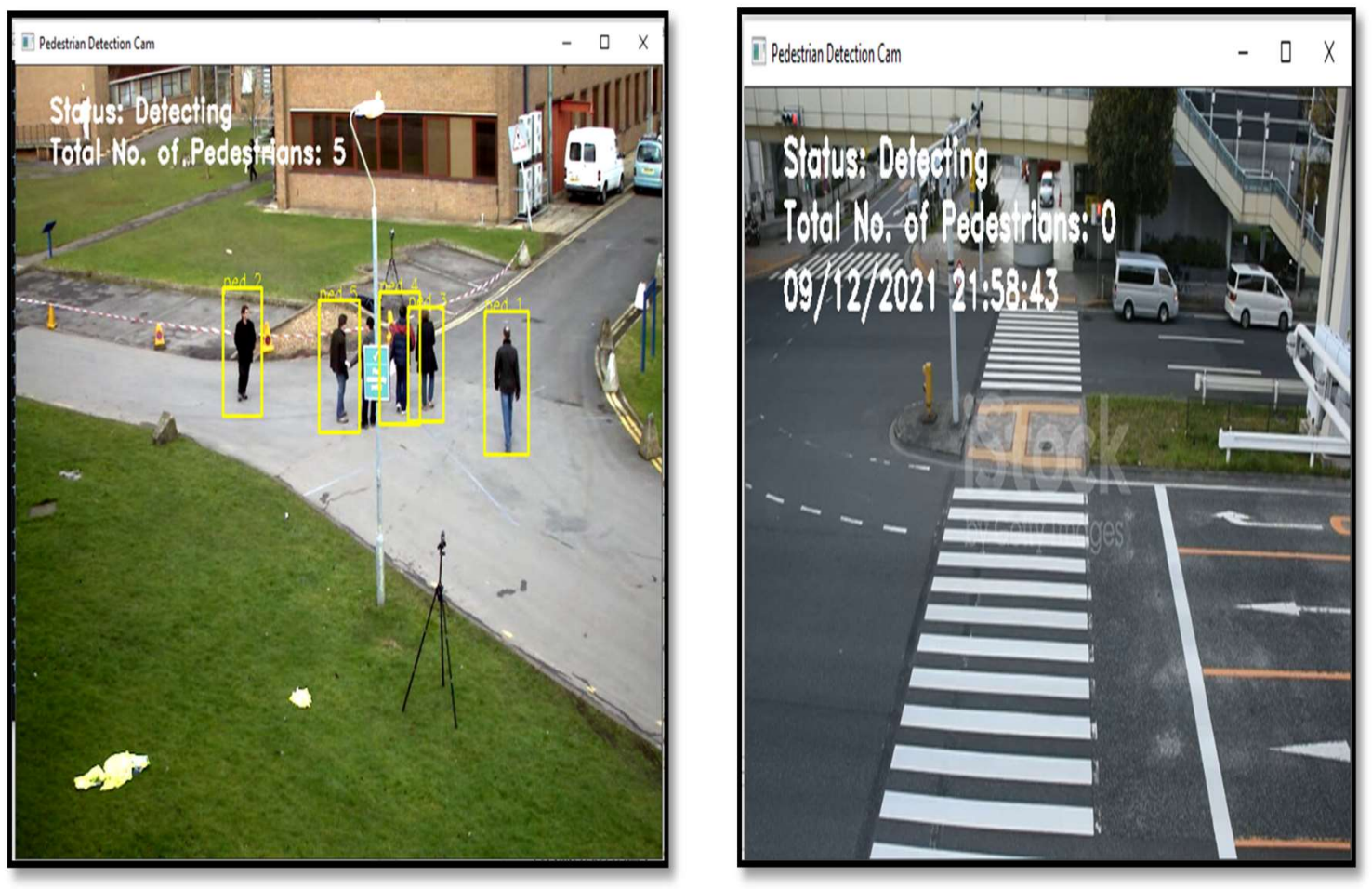

Fig. 2: Detection results using the pre-trained model acquired from the Haar Cascade Classifier 


\section{CONCLUDING REMARKS}

The Road Crossing Surveillance System is a Machine Learning Artificial Intelligence software that aid pedestrians to cross roads safely. The purpose of this project was to design, develop, and implement an autonomous road crossing and surveillance system that would help pedestrians cross roadways safely. The objective of this software is to reduce pedestrian waiting time and accidents when it comes to the crossing of roads.

This research is meant to arguably solve the problems of both the pedestrian crossing the road and the driver using the road. This is said to reduce waiting time for both drivers and pedestrians at the pedestrian crossing and if implemented well, will also reduce accidents on these pedestrian crossing 


\section{REFERENCES}

1. Bisandu, D. B. (2016). Design Science Research Methodology in Computer Science and Information Systems. International Journal of Information Technology, November 2016, 1-7.

2. Glèlè-ahanhanzo, Y., Kpozèhouen, A., Sossa-jerôme, C., Sopoh, G. E., Tedji, H., Yete, K., \& Levêque, A. (2021). "My right to walk, my right to live " : pedestrian fatalities, roads and environmental features in Benin. 2, 1-9.

3. Jing, P., Huang, W., \& Chen, L. (2017). Car-to-pedestrian communication safety system based on the vehicular Ad-Hoc network environment: A systematic review. In Information (Switzerland) (Vol. 8, Issue 4). https://doi.org/10.3390/info8040127

4. Levulytè, L., Baranyai, D., Sokolovskij, E., \& Török, Á. (2017). Pedestrians' Role in Road Accidents. International Journal for Traffic and Transport Engineering, 7(3), 328-341. https://doi.org/10.7708/ijtte.2017.7(3).04

5. Peffers, K., Tuunanen, T., Rothenberger, M. A., \& Chatterjee, S. (2007). A design science research methodology for information systems research. Journal of Management Information Systems, 24(3), 45-77. https://doi.org/10.2753/MIS0742-1222240302

6. Richey, R. C., \& Klein, J. D. (2014). Design and Development Research. In J. M. Spector, M. D. Merrill, J. Elen, \& M. J. Bishop (Eds.), Handbook of Research on Educational Communications and Technology (pp. 141-150). Springer New York. https://doi.org/10.1007/978-1-4614-3185-5_12 